\title{
Nonequilibrium steady states in fluids of platelike colloidal particles
}

\author{
Markus Bier* and René van Roij \\ Institute for Theoretical Physics, Utrecht University, Leuvenlaan 4, 3584CE Utrecht, The Netherlands \\ (Received 29 October 2007; published 5 February 2008; publisher error corrected 6 February 2008)
}

\begin{abstract}
Nonequilibrium steady states in an open system connecting two reservoirs of platelike colloidal particles are investigated by means of a recently proposed phenomenological dynamic density functional theory [M. Bier and R. van Roij, Phys. Rev. E 76, 021405 (2007)]. The platelike colloidal particles are approximated within the Zwanzig model of restricted orientations, which exhibits an isotropic-nematic bulk phase transition. Inhomogeneities of the local chemical potential generate a diffusion current which relaxes to a nonvanishing value if the two reservoirs coupled to the system sustain different chemical potentials. The relaxation process of initial states towards the steady state turns out to comprise two regimes: a smoothening of initial steplike structures followed by an ultimate relaxation of the slowest diffusive mode. The position of a nonequilibrium interface and the particle current of steady states depend nontrivially on the structure of the reservoirs due to the coupling between translational and orientational degrees of freedom of the fluid.
\end{abstract}

DOI: 10.1103/PhysRevE.77.021401

PACS number(s): 83.80.Hj, 61.20.Lc, 64.70.M-, 87.15.Vv

\section{INTRODUCTION}

Complex fluids of platelike colloidal particles, e.g., clay suspensions, have been investigated to quite an extent in recent years. The scientific interest largely stems from the enormous range of phenomena found in these systems such as flocculation, glass transitions, gelation, aging, and even liquid crystal phase transitions [1-13] due to the interplay of translational and orientational degrees of freedom of the constituting particles. Whereas the liquid crystal properties of fluids of platelike colloidal particles have been addressed in some theoretical studies devoted to homogeneous as well as inhomogeneous equilibrium systems [14-26], not many investigations of the nonequilibrium behavior have been performed.

In a recent publication the authors proposed a phenomenological dynamic density functional theory (DDFT) in order to describe the relaxation dynamics of fluids of platelike colloidal particles under the influence of an external field [27]. Relaxation into stable or metastable states, both characterized by a homogeneous local chemical potential, has been found depending on the initial state and the external field. In the present contribution, applying the same DDFT formalism, nonequilibrium steady states are investigated which form within a channel connecting two particle reservoirs of different chemical potentials. As the local chemical potential in the channel is expected to be spatially inhomogeneous DDFT can be considered as the natural formalism to describe the fluid of platelike colloidal particles on a coarsegrained level.

DDFT is an extension of equilibrium density functional theory (DFT) [28-30] to nonequilibrium conditions by proposing an equation of motion for the one-particle densities [31]. The one-particle densities are assumed to describe the (dynamical) state of the system completely. On the one hand, the DDFT equations are similar to the traditional timedependent Landau-Ginzburg and Cahn-Hillard models of

\footnotetext{
*m.bier@phys.uu.nl
}

critical dynamics, spinodal decomposition, and crystal growth [32-37]. On the other hand, the DDFT equations have been derived within the framework of (overdamped) Langevin dynamics, which is considered a reasonable description for dilute colloidal dispersions [38-41]. As the number densities of the present work are close to the isotropic-nematic two-phase region, which is located at small densities for highly anisotropic particles, the applied DDFT, which neglects hydrodynamic interactions, is expected to be valid. The DDFT proposed in Ref. [27] is briefly summarized in Sec. II.

The present work is restricted to fluids of platelike colloidal particles, i.e., nonergodic states such as glasses or gels are beyond the scope of this contribution. It is known that an ergodic system described by a linear master equation relaxes towards a unique steady state [42]. Although the formalism applied here is not equivalent to a linear master equation, the relaxation towards a unique steady state is nonetheless expected. This relaxation process is described in Sec. III in terms of the number density, the orientational order, the local chemical potential, and the particle current.

The final steady state is necessarily a nonequilibrium steady state because the particle reservoirs coupled to the system preclude equilibration of the system. This nonequilibrium steady state exhibits a nonvanishing particle current which is sustained by the chemical potential difference of the particle reservoirs coupled to the system. Moreover, for suitably chosen reservoir chemical potentials, the nonequilibrium steady state shows signs of bulk phase transitions. These issues are discussed in Sec. IV.

Section V discusses the results found in Secs. III and IV and closes with a short summary.

\section{FORMALISM}

\section{A. Model fluid and system geometry}

Consider a dispersion of monodisperse, hard, infinitely thin, square colloidal particles within a three-dimensional continuous solvent. The side length of the square particles is 
$D$. The orientations, described by the normal vector of the square face, are restricted to directions parallel to the Cartesian axes (Zwanzig approximation [43]). A particle is called an $i$ particle if its orientation is along the $i$ axis, $i \in\{x, y, z\}$.

The system under consideration is a channel of length $H$ which connects two particle reservoirs. The channel is assumed to be much wider than the particle size $D$ such that effects of the channel walls onto the colloidal fluid are negligible. Consequently, the fluid structure in the channel is expected to vary only along the channel axis which is taken as the $z$ axis. The channel is located at the $z$ axis interval $[0, H]$. The local number density of $i$ particles in the channel at position $z \in[0, H]$ is denoted by $\varrho_{i}(z)$. The abbreviation $\underline{\varrho}:=\left(\varrho_{x}, \varrho_{y}, \varrho_{z}\right)$ is used later.

The structure of the model fluid is adequately described in terms of the total density $\varrho:=\Sigma_{i} \varrho_{i}$ and the order parameter tensor $Q$ [44] which, within Zwanzig models, is given by

$$
Q_{i i^{\prime}}=\frac{1}{2}\left(3 \frac{\varrho_{i}}{\varrho}-1\right) \delta_{i i^{\prime}}
$$

where $\delta_{i i}$ is the Kronecker $\delta$.

The two-particle reservoirs are connected to the channel at positions $z=0$ and $z=H$, respectively. They are assumed to sustain equilibrium bulk structures $\underline{\varrho}_{0}$ and $\underline{\varrho}_{H}$ corresponding to the chemical potentials $\mu_{0}$ and $\mu_{H}$, respectively. The coupling of the two reservoirs to the system amounts to the Dirichlet-like boundary conditions $\underline{\varrho}(z \leqslant 0, t)=\underline{\varrho}_{0}$ and $\underline{\varrho}(z \geqslant H, t)=\underline{\varrho}_{H}$.

\section{B. Dynamic density functional theory}

Within this work the dynamic density functional theory (DDFT) proposed by the authors in Ref. [27] is applied. It consists of the set of equations of motion for the one-particle densities $\varrho_{i}$ given by

$$
\frac{\partial \varrho_{i}(z, t)}{\partial t}=\left(\frac{\partial \varrho_{i}(z, t)}{\partial t}\right)_{\text {trans }}+\left(\frac{\partial \varrho_{i}(z, t)}{\partial t}\right)_{\text {rot }},
$$

with the translational diffusion for fixed orientation described by

$$
\left(\frac{\partial \varrho_{i}(z, t)}{\partial t}\right)_{\text {trans }}:=-\frac{\partial j_{i}(z,[\underline{\varrho}(t)])}{\partial z},
$$

where the particle currents along the $z$ axis are

$$
j_{i}(z,[\varrho]):=-\Gamma_{i} \varrho_{i}(z) \frac{\partial \beta \mu_{i}(z,[\varrho])}{\partial z},
$$

and with the rotational diffusion at fixed position $z$ described by

$$
\begin{aligned}
\left(\frac{\partial \varrho_{i}(z, t)}{\partial t}\right)_{\text {rot }}:= & -\frac{1}{6 \tau} \sum_{i^{\prime}}\left[\varrho_{i}(z, t)+\varrho_{i^{\prime}}(z, t)\right] \\
& \times\left\{\beta \mu_{i}(z,[\underline{\varrho}(t)])-\beta \mu_{i^{\prime}}(z,[\underline{\varrho}(t)])\right\} .
\end{aligned}
$$

$[\varrho(t)]$ marks a quantity as a functional of all density profiles at time $t$. The driving force is due to inhomogeneities of the local chemical potential

$$
\mu_{i}(z,[\underline{\varrho}]):=\left.\frac{\delta F}{\delta \varrho_{i}(z)}\right|_{\underline{\varrho}},
$$

which derives from the free energy functional $[45,46]$

$$
\beta F[\underline{\varrho}]=\int d z\left(\sum_{i} \varrho_{i}(z)\left[\ln \left(\varrho_{i}(z) \Lambda^{3}\right)-1\right]+\Phi(\underline{n}(z))\right),
$$

where

$$
\Phi(\underline{n}(z))=n_{0}(z) \ln \left[1-n_{3}(z)\right]+\frac{\sum_{q} n_{1 q}(z) n_{2 q}(z)}{1-n_{3}(z)}+\frac{\prod_{q} n_{2 q}(z)}{\left[1-n_{3}(z)\right]^{2}}
$$

describes the excess free energy density due to the hard-core interaction. The weighted densities $n_{\alpha}(z):=\sum_{i} \omega_{\alpha, i} \otimes \varrho_{i}(z)$ involve the convolution $(\otimes)$ of the densities $\varrho_{i}$ with the weight functions

$$
\begin{gathered}
\omega_{0, i}(z)=a\left(z, S_{z i}\right), \quad \omega_{1 x, i}(z)=S_{x i} a\left(z, S_{z i}\right), \\
\omega_{1 y, i}(z)=S_{y i} a\left(z, S_{z i}\right), \quad \omega_{1 z, i}(z)=b\left(z, S_{z i}\right), \\
\omega_{2 x, i}(z)=S_{y i} b\left(z, S_{z i}\right), \quad \omega_{2 y, i}(z)=S_{x i} b\left(z, S_{z i}\right), \\
\omega_{2 z, i}(z)=S_{x i} S_{y i} a\left(z, S_{z i}\right), \quad \omega_{3, i}(z)=S_{x i} S_{y i} b\left(z, S_{z i}\right),
\end{gathered}
$$

where the abbreviations $a(z, S):=\frac{1}{2}\left[\delta\left(\frac{S}{2}+z\right)+\delta\left(\frac{S}{2}-z\right)\right]$ and $b(z, S):=\Theta\left(\frac{S}{2}-|z|\right)$ are used and $S_{q i} \in\{0, D\}$ denotes the extension of $i$ particles along the $q$ axis. The rotational relaxation time

$$
\tau=\frac{2}{9} \beta \eta D^{3}
$$

and the translational diffusion constants

$$
\Gamma_{x, y}=\frac{D^{2}}{24 \tau}, \quad \Gamma_{z}=\frac{D^{2}}{36 \tau}
$$

have been chosen [47]. In the above equations $\beta$ is the inverse temperature, $\Lambda$ denotes the thermal de Broglie wavelength, and $\eta$ is the viscosity of the solvent.

The described DDFT neglects hydrodynamic interactions between the platelike particles. This is considered as a reasonable approximation because of the small particle densities in this study close to the bulk isotropic-nematic two-phase coexistence region $[48,49]$.

An equilibrium state $\underline{\varrho}^{\mathrm{eq}}$ fulfills the Euler-Lagrange equation

$$
\mu_{i}\left(z,\left[\underline{\varrho}^{\mathrm{eq}}\right]\right)=\mu,
$$

with the chemical potential $\mu$, i.e., equilibrium density profiles render the local chemical potential as a function of position $(z)$ and orientation $(i)$ into a constant. Therefore the described DDFT is consistent with equilibrium DFT because any equilibrium state $\varrho^{\text {eq }}$ is stationary under the dynamics represented by Eqs. (2)-(5). Moreover, from Eqs. (4) and 

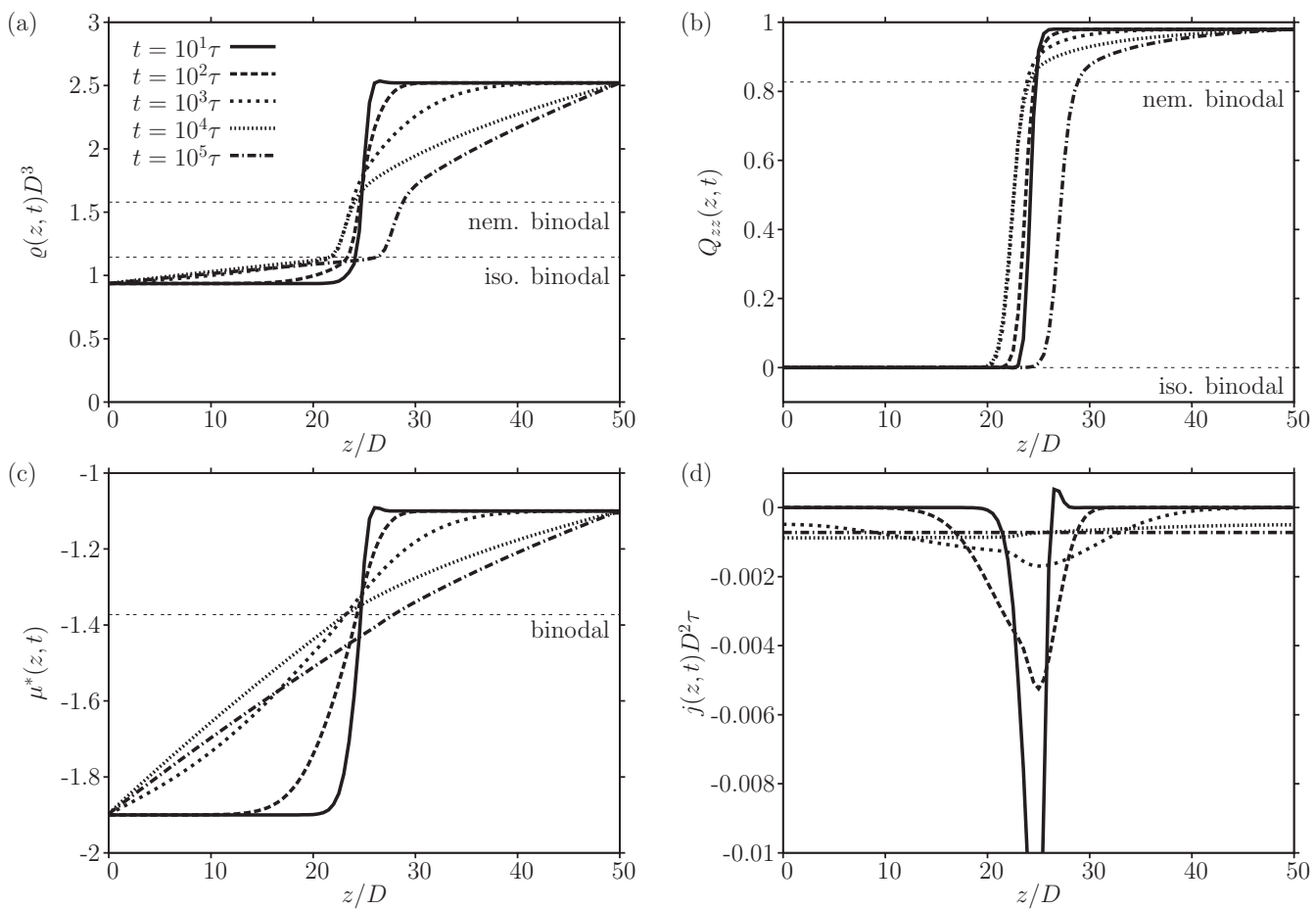

FIG. 1. (a) Total number density $\varrho(z, t)$, (b) nematic order paramter tensor component parallel to the $z$ axis $Q_{z z}(z, t)$, (c) reduced local chemical potential $\mu^{*}(z, t)$, and (d) total particle current $j(z, t)$ of platelike colloidal particles of size $D$ in a channel of length $H=50 D$ connecting two particle reservoirs which sustain bulk equilibrium structures corresponding to the reduced chemical potentials $\mu_{0}^{*}=-1.9$ at $z=0$ and $\mu_{H}^{*}=-1.1$ at $z=H$. The structure in the reservoir at $z=0$ is isotropic whereas the structure in the reservoir at $z=H$ is nematic with the director parallel to the $z$ axis. The isotropic and the nematic binodals of the colloidal fluid in equilibrium are displayed for comparison. The time is given in terms of the rotational relaxation time $\tau$. No further changes are visible for times $t \geqslant 10^{5} \tau$.

(12) one concludes a vanishing particle current for equilibrium states: $j\left[\varrho^{\mathrm{eq}}\right]=0$. For later convenience, the reduced (local) chemical potential $\mu^{*}:=\beta \mu-3 \ln \left(\frac{2 \Lambda}{D}\right)$ is defined.

\section{Bulk phase behavior and numerical method}

The described model of fluids of platelike colloidal particles exhibits a first-order isotropic-nematic bulk phase transition at a reduced chemical potential $\mu_{\mathrm{b}}^{*}=-1.3727$. At this binodal $(b)$ the isotropic bulk phase of density $\varrho_{\mathrm{b}}^{\text {iso }} D^{3}$ $=1.1440$ coexists with the nematic bulk phase of density $\varrho_{\mathrm{b}}^{\text {nem }} D^{3}=1.5789$ and scalar order parameter $S_{\mathrm{b}}^{\text {nem }}$ $=\left\langle\frac{3}{2} \cos (\vartheta)^{2}-\frac{1}{2}\right\rangle=0.82696$, where \langle\rangle denotes the thermal average and $\vartheta$ is the angle between the particle orientation and the director [44]. Solutions of the DDFT equation specified in Sec. II B are calculated numerically by means of the Euler-forward method with integration time steps of $\frac{\tau}{20}$.

\section{RELAXATION TOWARDS THE STEADY STATE}

In this section the relaxation process of an initial state $\varrho(t=0)$ towards the stationary state corresponding to the boundary conditions exerted by the two reservoirs coupled to the system at $z=0$ and $z=H$ (see Sec. II) is studied. Here the arbitrary case of the reservoir at $z=0$ sustaining the isotropic bulk structure $\underline{\varrho}_{0}$ corresponding to the reduced chemical potential $\mu_{0}^{*}=-1.9$ and the reservoir at $z=H$ sustaining the nematic bulk structure with the director parallel to the $z$ axis $\underline{\varrho}_{H}$ corresponding to $\mu_{H}^{*}=-1.1$ is described in detail. Moreover, the initial state discussed here is chosen as

$$
\underline{\varrho}(z, t=0)=\left\{\begin{array}{l}
\varrho_{H}, \quad z>\frac{H}{2}, \\
\underline{\varrho}_{0}, \quad z<\frac{H}{2},
\end{array}\right.
$$

which approximates the equilibrium structure in the channel in the presence of an impermeable membrane located at $z$ $=\frac{H}{2}$. Here a detailed discussion of the case of the nematic state $\underline{\varrho}_{H}$ with the director perpendicular to the $z$ axis is not necessary, because the temporal evolution turns out to be qualitatively the same as for the case of parallel alignment.

The solutions of the DDFT equations (2)-(11) have also been calculated for different initial states than in Eq. (13), such as the linear density profile $\underline{\varrho}(0 \leqslant z \leqslant H, t=0)$ $=\left(1-\frac{z}{H}\right) \underline{\varrho}_{0}+\frac{z}{H} \varrho_{H}$ and the almost empty channel $\underline{\varrho}(0 \leqslant z \leqslant H, t=0) \approx 0$, in order to verify that the final steady state is independent of the initial state.

In Fig. 1 the temporal evolution of the initial state equation (13) for the channel length $H=50 D$ is displayed. The total number density $\varrho(z, t)$ is shown in Fig. 1(a). Up to time $t \lesssim 10^{4} \tau$ the initial steplike density profile smoothens by diffusion until the whole channel at $z \in[0, H]$ is affected. $\varrho(z, t)$ for times $t \geqslant 10^{5} \tau$ does not undergo visible changes, i.e., 
from then on the steady state is (practically) attained. The equilibrium bulk density values of the isotropic binodal $\varrho_{\mathrm{b}}^{\text {iso }}$ and the nematic binodal $\varrho_{\mathrm{b}}^{\text {nem }}$ are shown in Fig. 1(a), too. The steady state is approximately linear below the isotropic and above the nematic binodal densities. In the density range between the binodals, corresponding to the bulk two-phase coexistence region, a steep portion of the steady state density profile is related to the isotropic-nematic bulk phase transition. It has been verified by solving the DDFT equations that in the case of two reservoirs of both isotropic structure a similar steep portion is absent.

Figure 1(b) displays the temporal evolution of the nematic order parameter tensor component parallel to the $z$ axis $Q_{z z}(z, t)$. Similar to the total number density profile $\varrho(z, t)$ in Fig. 1(a), a diffusive smoothening of the initially steplike profile takes place at times $t \lesssim 10^{4} \tau$, whereas the steady state has been attained at times $t \geqslant 10^{5} \tau$. Between the order parameter values at the isotropic binodal 0 and the nematic binodal $S_{\mathrm{b}}^{\text {nem }}$ the order parameter profile increases rapidly as a function of position.

For times larger than the rotational relaxation time, $t \gg \tau$, the local chemical potentials $\mu_{i}^{*}$ for $i$ particles are practically independent of $i$. Hence it is useful to consider the reduced chemical potential profile $\mu^{*}(z, t):=\frac{1}{3} \sum_{i} \mu_{i}^{*}(z, t)$, which is depicted in Fig. 1(c). Again a smoothening of the initial steplike profile in the time range $t \lesssim 10^{4} \tau$ due to diffusion is followed by a restructuring into the final steady state which has been reached at times $t \geqslant 10^{5} \tau$. The slope of the steady state profile as a function of position $z$ slightly decreases upon increasing $z$.

In Fig. 1(d) the total particle current $j(z, t):=\Sigma_{i} j_{i}(z, t)$ is displayed. At early times the current is localized near the position $z=\frac{H}{2}$ of the discontinuity of the initial state equation (13). Because $\mu_{0}^{*}<\mu_{H}^{*}$ the current is negative. With time the spatial current distribution $j(z, t)$ broadens and ultimately becomes homogeneous in the steady state. Under the present conditions (reservoir chemical potentials, nematic director alignment, channel length) the steady state current $j(z, t=\infty) D^{2} \tau=-7.219 \times 10^{-4}$ is attained.

In order to quantify the "distance" of a given state $\varrho$ from the steady state, which is characterized by a homogeneous particle current, consider the nonstationarity parameter

$$
\varepsilon[\underline{\varrho}]:=\max _{z \in[0, H]} j(z,[\underline{\varrho}])-\min _{z \in[0, H]} j(z,[\underline{\varrho}]),
$$

which is a functional of the state $\underline{Q}$. Obviously $\varepsilon[\varrho] \geqslant 0$. The nonstationarity parameter vanishes if and only if the current is homogeneous, e.g., if $\varrho$ is a steady state.

Figure 2 depicts the nonstationarity parameter $\varepsilon[\underline{Q}(t)]$ evaluated for the state $\underline{\varrho}(t)$ evolved from the initial state equation (13) as a function of time $t$ for channel lengths $H$ $=100 \mathrm{D}$ (solid line), $H=50 \mathrm{D}$ (dashed line), and $H=25 \mathrm{D}$ (dotted line). In Fig. 2(a) one identifies a power law behavior $\varepsilon$ $\sim t^{-1 / 2}$ within a time range $\tau \leqslant t \lesssim t^{\times}(H)$, where $t^{\times}(H)$ denotes an (approximate) crossover time, which depends on the channel length $H$. The exponent is a consequence of the diffusive smoothening (see Fig. 1) of the initial steplike chemical potential profile: If $d(t)$ denotes the "width" of the inter-
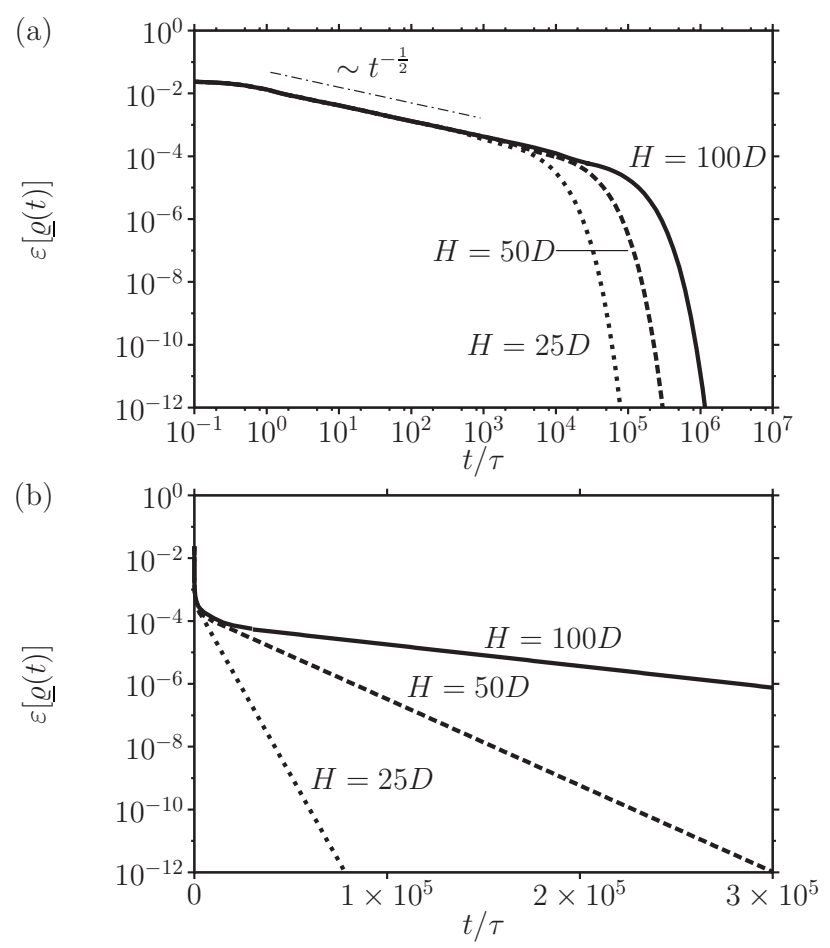

FIG. 2. Nonstationarity parameter $\varepsilon[\underline{\varrho}(t)]$, defined in the main text, of state $\underline{Q}(t)$ at time $t$ for channel lengths $H=100 D$ (solid line), $H=50 D$ (dashed line), and $H=25 D$ (dotted line). The time is given in terms of the rotational relaxation time $\tau$. In the time range $\tau$ $\leqslant t \lesssim t^{\times}(H)$ with $t^{\times}(H) \sim H^{2}$, diffusive smoothening of the initial steplike state leads to a power law behavior $\varepsilon \sim t^{-1 / 2}$ [see (a)]. At times $t \approx t^{\times}(H)$, a crossover to an ultimately exponential decay of $\varepsilon$ [see (b)] takes place. The time scale of this exponential decay is proportional to $H^{2}$.

val in Fig. 1(c) where $\mu^{*}(z, t)$ deviates considerably from the reservoir values one expects a diffusive power law $d(t)$ $\sim t^{1 / 2}$ as long as $d(t)<H$. Since the particle current $j(z, t)$ is determined by the gradient of the local chemical potential [see Eq. (4)], which in the smoothened interval is approximately given by $\frac{\mu_{H}^{*}-\mu_{0}^{*}}{d(t)}$, one finds $\varepsilon[\varrho(t)] \sim d(t)^{-1} \sim t^{-1 / 2}$. The crossover time $t^{\times}(H)$, which marks the end of the diffusive smoothening process, could be defined by $d\left(t^{\times}(H)\right)=H$, i.e., one expects, $t^{\times}(H) \sim H^{2}$. At times $t \gtrsim t^{\times}(H)$ an ultimate exponential decay of $\varepsilon[\underline{\varrho}(t)]$ is found [Fig. 2(b)]. The decay times $\tau^{*}(H)$ of this exponential decay are $\tau^{*}(100 D)$ $=63260 \tau, \tau^{*}(50 D)=15793 \tau$, and $\tau^{*}(25 D)=3959 \tau$ for the reservoir configurations discussed in the present section. These values are proportional to $H^{2}$ and they are of the same order of magnitude as $\frac{H^{2}}{2 \Gamma_{x, y, z}}$, the one-dimensional translational diffusion time which is expected to be related to the ultimate relaxation time scale.

\section{PROPERTIES OF THE STEADY STATE}

In the previous section one specific set of reservoir configurations has been fixed arbitrarily in order to study general features of the temporal relaxation towards the correspond- 
ing stationary state. Here only properties of stationary states and their dependence on the reservoir configurations is studied. However, the investigation is restricted to cases with one reservoir sustaining an isotropic structure $\varrho_{0}$ at $z=0$ and one reservoir sustaining a nematic structure $\varrho_{H}$ at $z=H$. In contrast to the previous section both possible alignments of the nematic director with respect to the $z$ axis-parallel and perpendicular-are considered explicitly. We focus on the isotropic-nematic interface and on the magnitude of the particle current as functions of the boundary conditions set by the reservoirs.

As a general qualitative feature, revealed in the previous section, the stationary state varies rapidly with position if it is "close" to the (equilibrium) bulk two-phase coexistence region [see profiles $t=10^{5} \tau$ in Figs. 1(a) and 1(b)]. This feature is reminiscent of a free interface between an isotropic and a nematic phase at equilibrium bulk coexistence. Here, however, the steplike profiles are nonequilibrium structures; we call them nonequilibrium interfaces. There are several possibilities to describe the "position" $z$ int of the nonequilibrium interface. Here the following three are considered:

$$
\begin{gathered}
\varrho\left(z_{\varrho}^{\mathrm{int}}, t=\infty\right):=\frac{1}{2}\left(\varrho_{\mathrm{b}}^{\mathrm{iso}}+\varrho_{\mathrm{b}}^{\mathrm{nem}}\right), \\
\left(\max _{i} Q_{i i}\right)\left(z_{Q}^{\mathrm{int}}, t=\infty\right):=S_{\mathrm{b}}^{\mathrm{nem}}, \\
\mu^{*}\left(z_{\mu}^{\mathrm{int}}, t=\infty\right):=\mu_{\mathrm{b}}^{*} .
\end{gathered}
$$

The different definitions of the interface position $z^{\text {int }}$ are displayed in Fig. 3 for a channel of length $H=50 D$ and for different alignments of the nematic director of the reservoir state $\underline{\varrho}_{H}$. They are ordered as $z_{\varrho}^{\text {int }}<z_{\mu}^{\text {int }}<z_{Q}^{\text {int }}$ with $z_{\mu}^{\text {int }}-z_{\varrho}^{\text {int }}$ $\approx 0.1 D$. Moreover, $z_{Q}^{\text {int }}-z_{\varrho}^{\text {int }} \approx 0.3 D$ for parallel and $z_{Q}^{\text {int }}-z_{Q}^{\text {int }}$ $\approx 0.6 \mathrm{D}$ for perpendicular alignment of the nematic director. These differences compared to the channel length are small, however, hence one concludes that the three definitions for the interface position equation (15) are equally reasonable.

Figure 3(a) depicts the interface position $z^{\text {int }}$ as a function of the density in the nematic reservoir $\varrho_{H}$ with the density in the isotropic reservoir fixed to $\varrho_{0} D^{3}=\varrho_{\mathrm{b}}^{\text {iso }} D^{3}-0.04$. Upon increasing the nematic reservoir density $\varrho_{H}$ the nonequilibrium interface is shifted towards the isotropic reservoir $(z=0)$. Conversely, Fig. 3(b) displays the interface position $z^{\text {int }}$ as a function of the isotropic reservoir density $\varrho_{0}$ where the nematic reservoir density is fixed to $\varrho_{H} D^{3}=\varrho_{\mathrm{b}}^{\text {nem }} D^{3}+0.4$. Under these conditions, the nonequilibrium interface shifts towards the nematic reservoir $(z=H)$ upon decreasing the density of the isotropic reservoir $\varrho_{0}$. Moreover, for given reservoir densities, the nonequilibrium interface for parallel alignment of the nematic director is located closer to the nematic reservoir $(z=H)$ than for perpendicular alignment.

The dependence of the total current of the stationary state $j(t=\infty)$ on the configurations of the reservoirs is displayed in Fig. 4. In Fig. 4(a) the isotropic reservoir density is fixed to $\varrho_{0} D^{3}=\varrho_{\mathrm{b}}^{\text {iso }} D^{3}-0.04$, whereas in Fig. 4(b) the nematic reservoir density is fixed to $\varrho_{H} D^{3}=\varrho_{\mathrm{b}}^{\text {nem }} D^{3}+0.4$. The main plots exhibit $j(t=\infty)$ as a function of the reservoir densities $\varrho_{H}$
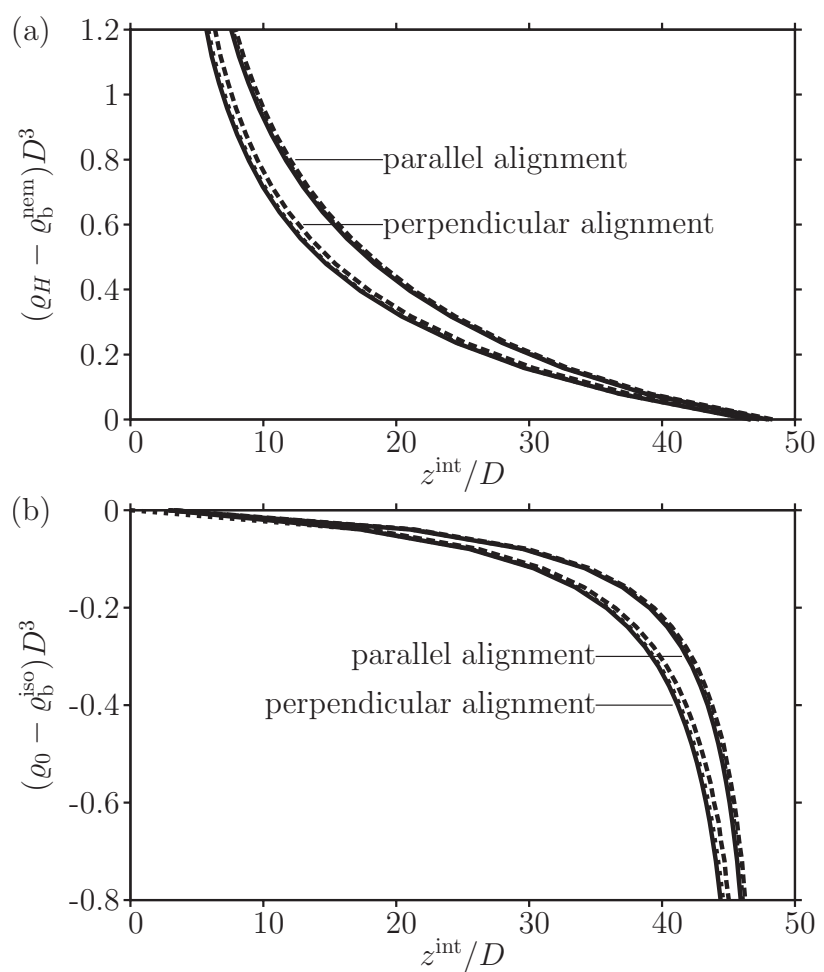

FIG. 3. Position of the nonequilibrium isotropic-nematic interface $z^{\text {int }}$ in a channel of length $H=50 D$ with the nematic director aligned parallel and perpendicular to the $z$ axis. The curves corresponding to the interface postitions $z_{\varrho}^{\text {int }}$ (solid line), $z_{Q}^{\text {int }}$ (dashed line), and $z_{\mu}^{\text {int }}$ (dotted line) defined in the main text almost coincide. In (a) the isotropic reservoir is fixed to $\left(\varrho_{0}-\varrho_{b}^{\text {iso }}\right) D^{3}=-0.04$, whereas in (b) the nematic reservoir is fixed to $\left(\varrho_{H}-\varrho_{b}^{\text {nem }}\right) D^{3}=0.4$.

[Fig. 4(a)] and $\varrho_{0}$ [Fig. 4(b)]; the insets alternatively display $j(t=\infty)$ as a function of the chemical potential difference of the reservoirs $\mu_{H}^{*}-\mu_{0}^{*}$. Since $\varrho_{H}>\varrho_{0}$, or equivalently $\mu_{H}^{*}$ $>\mu_{0}^{*}$, the total current is negative. The magnitude $|j(t=\infty)|$ increases with $\varrho_{H}-\varrho_{0}$ and it is larger for perpendicular than for parallel alignment of the nematic director. The latter observation is not surprising if one recalls $\Gamma_{x, y}>\Gamma_{z}$ [Eq. (11)]. However, the two cases shown in Figs. 4(a) and 4(b) are remarkably different in the sense that the current difference between perpendicular and parallel alignment $\left|j_{\perp}(t=\infty)\right|$ $-\left|j_{\|}(t=\infty)\right|$ is almost constant upon fixing the nematic reservoir density $\varrho_{H}$ and varying the isotropic reservoir density $\varrho_{0}$ [Fig. 4(b)], whereas this difference increases upon increasing the nematic reservoir density $\varrho_{H}$ and fixing the isotropic reservoir density $\varrho_{0}$ [Fig. 4(a)]. Finally, for the same reservoir density difference $\varrho_{H}-\varrho_{0}$, the magnitude of the stationary state current $|j(t=\infty)|$ in Fig. 4(b) where most of the channel is filled with isotropic fluid is larger than in Fig. 4(a) where the channel contains predominantly nematic fluid.

\section{DISCUSSION AND SUMMARY}

In the present work nonequilibrium steady states of a fluid of platelike colloidal particles in a channel which connects two reservoirs sustaining bulk structures of different chemi- 

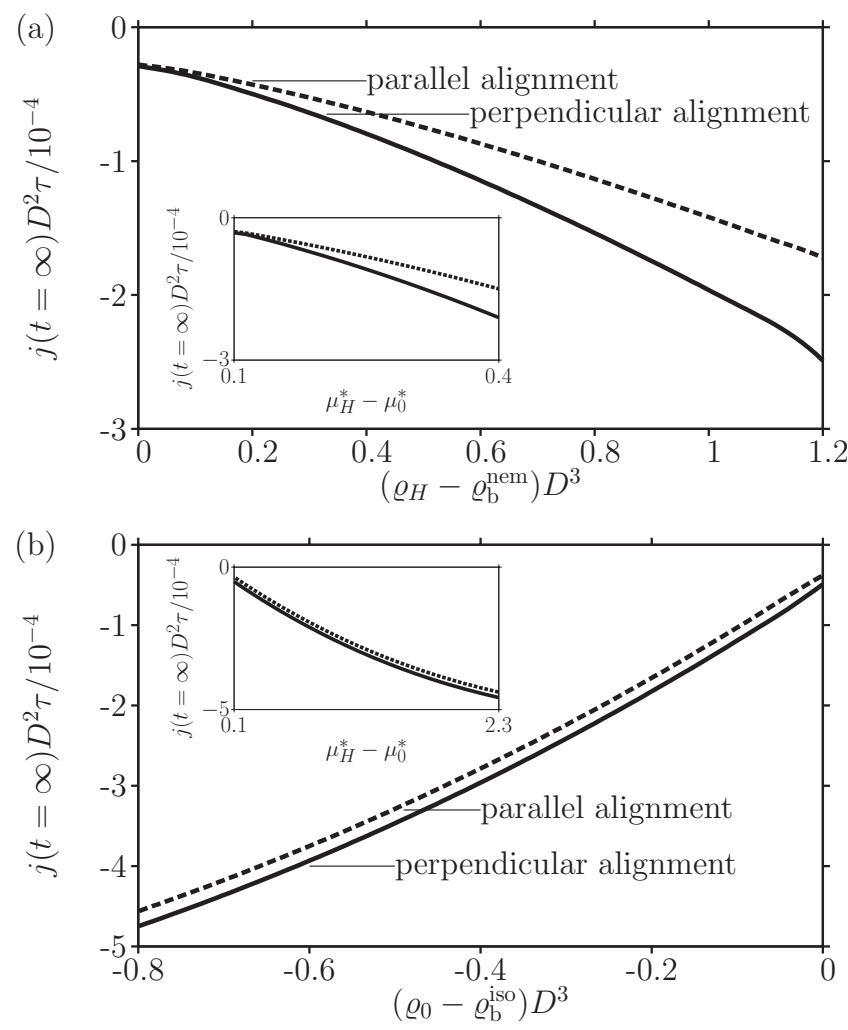

FIG. 4. Stationary state current $j(t=\infty)$ in a channel of length $H=50 D$ with the nematic director aligned parallel and perpendicular to the $z$ axis. In (a) the isotropic reservoir density is fixed to $\left(\varrho_{0}-\varrho_{b}^{\text {iso }}\right) D^{3}=-0.04$, whereas in (b) the nematic reservoir density is fixed to $\left(\varrho_{H}-\varrho_{b}^{\text {nem }}\right) D^{3}=0.4$. The main plots exhibit $j(t=\infty)$ as a function of (a) $\varrho_{H}$ and (b) $\varrho_{0}$. An alternative representation of $j(t=\infty)$ as a function of the chemical potential difference of the reservoirs $\mu_{H}^{*}-\mu_{0}^{*}$ is displayed in the insets.

cal potentials have been investigated. A typical platelet fluid is sample A10P of Ref. [50], which consists of an aqueous dispersion (solvent viscosity $\eta=8.9 \times 10^{-4} \mathrm{~Pa} \mathrm{~s}$ ) of sterically stabilized gibbsite platelets of diameter $D=165 \mathrm{~nm}$. According to Eq. (10) the rotational relaxation time is given by $\tau$ $\approx 214 \mu \mathrm{s}$. The calculated translational diffusion coefficients [see Eq. (11)] compare well with the measured ones of Ref. [50]. The numerical results of Sec. III suggest that the fluid in a channel of length $H=50 D \approx 8.3 \mu \mathrm{m}$ has definitely reached the steady state within a time $t=10^{5} \tau \approx 21 \mathrm{~s}$. The corresponding steady state current is $|j(t=\infty)| D^{2}=7.219$ $\times 10^{-4} / \tau \approx 3.4 \mathrm{~s}^{-1}$. In this work the channel width is assumed to be much larger than the particle size such that effects of the channel walls can be neglected. For a channel width of $10 \mu \mathrm{m} \approx 61 D$, say, a steady state channel current of approximately 12400 platelets per second is found. As a reservoir of volume $1 \ell=0.001 \mathrm{~m}^{3}$ contains of the order of $10^{17}$ platelike particles, the reservoir density in an experimental realization of the setting discussed here stays constant over any conceivable experimental time scale.

It has been shown in Sec. III that the relaxation process of the platelet fluid towards the steady state is purely diffusive. It comprises a diffusive smoothening of the initial steplike fluid structure until the complete channel is affected, which takes a time proportional to $H^{2}$, followed by a structural relaxation corresponding to the slowest diffusion mode with a relaxation time proportional to $H^{2}$. The two different regimes have been detected by a power law and an exponential decay, respectively, of the nonstationarity parameter $\varepsilon$ [Eq. (14)], which measures the inhomogeneity of the particle current within the channel. In light of the rather complicated nonlocal fluid model (Sec. II) involving translational as well as orientational degrees of freedom, the clear identification of the power law and exponential decay regimes of $\varepsilon$ as a function of time suggests that these regimes might also be easily found in real platelet fluids.

In summary, the present work studies the formation and the structure of nonequilibrium steady states in fluids of platelike colloidal particles in a channel by means of dynamic density functional theory. Localized rapid changes of density [Fig. 1(a)] and order parameter tensor [Fig. 1(b)] profiles of nonequilibrium steady states are similar to free interfaces. The local chemical potential profile interpolates smoothly between the values sustained by reservoirs at the ends of the channel [Fig. 1(c)]. The broadening of the particle current distribution [Fig. 1(d)] suggests the introduction of a parameter measuring the distance from the steady state. The purely diffusive relaxation process towards the steady state comprises two regimes: a smoothening of the initial steplike structure followed by an ultimate relaxation of the slowest diffusive mode (Fig. 2). The position of a nonequilibrium interface (Fig. 3) and the particle current (Fig. 4) of steady states depend nontrivially on the structure of the reservoirs due to the coupling between translational and orientational degrees of freedom of the fluid.

\section{ACKNOWLEDGMENTS}

The authors thank Marjolein Dijkstra for access to additional computational resources. This work is part of the research program of the "Stichting voor Fundamenteel Onderzoek der Materie (FOM)," which is financially supported by the "Nederlandse Organisatie voor Wetenschappelijk Onderzoek (NWO)."
[1] A. Mourchid, A. Delville, J. Lambard, E. Lécolier, and P. Levitz, Langmuir 11, 1942 (1995).

[2] A. B. D. Brown, S. M. Clarke, and A. R. Rennie, Langmuir 14, 3129 (1998).

[3] A. Mourchid, E. Lécolier, H. van Damme, and P. Levitz, Lang- muir 14, 4718 (1998).

[4] F. M. van der Kooij and H. N. W. Lekkerkerker, J. Phys. Chem. B 102, 7829 (1998).

[5] D. Bonn, H. Kellay, H. Tanaka, G. Wegdam, and J. Meunier, Langmuir 15, 7534 (1999). 
[6] A. B. D. Brown, C. Ferrero, T. Narayanan, and A. R. Rennie, Eur. Phys. J. B 11, 481 (1999).

[7] P. Levitz, E. Lécolier, A. Mourchid, A. Delville, and S. Lyonnard, Europhys. Lett. 49, 672 (2000).

[8] A. Knaebel, M. Bellour, M.-P. Munch, V. Viasnoff, F. Lequeux, and J. L. Harden, Europhys. Lett. 52, 73 (2000).

[9] B. Abou, D. Bonn, and J. Meunier, Phys. Rev. E 64, 021510 (2001).

[10] D. van der Beek and H. N. W. Lekkerkerker, Europhys. Lett. 61, 702 (2003).

[11] S. Liu, J. Zhang, N. Wang, W. Liu, C. Zhang, and D. Sun, Chem. Mater. 15, 3240 (2003).

[12] D. van der Beek and H. N. W. Lekkerkerker, Langmuir 20, 8582 (2004).

[13] N. Wang, S. Liu, J. Zhang, Z. Wu, J. Chen, and D. Sun, Soft Matter 1, 428 (2005).

[14] J. A. Cuesta and R. P. Sear, Eur. Phys. J. B 8, 233 (1999).

[15] D. G. Rowan and J.-P. Hansen, Langmuir 18, 2063 (2002).

[16] L. Harnau, D. Costa, and J.-P. Hansen, Europhys. Lett. 53, 729 (2001).

[17] L. Harnau and S. Dietrich, Phys. Rev. E 65, 021505 (2002).

[18] L. Harnau, D. Rowan, and J.-P. Hansen, J. Chem. Phys. 117, 11359 (2002).

[19] M. Bier, L. Harnau, and S. Dietrich, Phys. Rev. E 69, 021506 (2004).

[20] L. Harnau and S. Dietrich, Phys. Rev. E 69, 051501 (2004).

[21] D. Costa, J.-P. Hansen, and L. Harnau, Mol. Phys. 103, 1917 (2005).

[22] L. Harnau and S. Dietrich, Phys. Rev. E 71, 011504 (2005).

[23] M. Bier, L. Harnau, and S. Dietrich, J. Chem. Phys. 123, 114906 (2005).

[24] M. Bier, L. Harnau, and S. Dietrich, J. Chem. Phys. 125, 184704 (2006).

[25] D. van der Beek, H. Reich, P. van der Schoot, M. Dijkstra, T. Schilling, R. Vink, M. Schmidt, R. van Roij, and H. Lekkerkerker, Phys. Rev. Lett. 97, 087801 (2006).

[26] H. Reich, M. Dijkstra, R. van Roij, and M. Schmidt, J. Phys. Chem. B 111, 7825 (2007).

[27] M. Bier and R. van Roij, Phys. Rev. E 76, 021405 (2007).

[28] R. Evans, Adv. Phys. 28, 143 (1979).

[29] R. Evans, in Liquides aux Interfaces/Liquids at Interfaces, ed- ited by J. Charvolin, J. F. Joanny, and J. Zinn-Justin, Proceedings of Les Houches, Session XLVIII, 1988 (North-Holland, Amsterdam, 1989), p. 1.

[30] R. Evans, in Inhomogeneous Fluids, edited by D. Henderson (Dekker, New York, 1991), p. 89.

[31] W. Dieterich, H. L. Frisch, and A. Majhofer, Z. Phys. B: Condens. Matter 78, 317 (1990).

[32] J. S. Langer, Ann. Phys. 65, 53 (1971).

[33] K. Kawasaki, Prog. Theor. Phys. 57, 410 (1977).

[34] J. B. Collins and H. Levine, Phys. Rev. B 31, 6119 (1985); 33, 2020(E) (1986).

[35] P. R. Harrowell and D. W. Oxtoby, J. Chem. Phys. 86, 2932 (1987).

[36] W. J. Boettinger, J. A. Warren, C. Beckermann, and A. Karma, Annu. Rev. Mater. Res. 32, 163 (2002).

[37] L. Gránásy, T. Pusztai, and T. Börzsönyi, in Handbook of Theoretical and Computational Nanotechnology, edited by $\mathrm{M}$. Rieth and W. Schommers (American Scientific Publishers, Stevenson Ranch, 2006), Vol. 9, p. 525.

[38] D. S. Dean, J. Phys. A 29, L613 (1996).

[39] U. Marini Bettolo Marconi and P. Tarazona, J. Chem. Phys. 110, 8032 (1999).

[40] U. Marini Bettolo Marconi and P. Tarazona, J. Phys.: Condens. Matter 12, A413 (2000).

[41] A. J. Archer and R. Evans, J. Chem. Phys. 121, 4246 (2004).

[42] J. Schnakenberg, Rev. Mod. Phys. 48, 571 (1976).

[43] R. Zwanzig, J. Chem. Phys. 39, 1714 (1963).

[44] P. G. de Gennes and J. Prost, The Physics of Liquid Crystals (Oxford University Press, Oxford, 1993).

[45] J. A. Cuesta and Y. Martínez-Ratón, Phys. Rev. Lett. 78, 3681 (1997).

[46] J. A. Cuesta and Y. Martínez-Ratón, J. Chem. Phys. 107, 6379 (1997).

[47] H. Brenner, Int. J. Multiphase Flow 1, 195 (1974).

[48] X. Qiu, X. L. Wu, J. Z. Xue, D. J. Pine, D. A. Weitz, and P. M. Chaikin, Phys. Rev. Lett. 65, 516 (1990).

[49] J.-Z. Xue, X.-L. Wu, D. J. Pine, and P. M. Chaikin, Phys. Rev. A 45, 989 (1992).

[50] F. M. van der Kooij, A. P. Philipse, and J. K. G. Dhont, Langmuir 16, 5317 (2000). 